\title{
What we know so far: COVID-19 current clinical knowledge and research
}

\author{
Author: Mary A Lake ${ }^{\mathrm{A}}$
}

In December 2019, health authorities in Wuhan, China, identified a cluster of pneumonia cases of unknown aetiology linked to the city's South China Seafood Market. Subsequent investigations revealed a novel coronavirus, SARS-CoV-2, as the causative agent now at the heart of a major outbreak. The rising case numbers have been accompanied by unprecedented public health action, including the wholesale isolation of Wuhan. Alongside this has been a robust scientific response, including early publication of the pathogen genome, and rapid development of highly specific diagnostics. This article will review the new knowledge of SARS-CoV-2 COVID-19 acute respiratory disease, and summarise its clinical features.

KEYWORDS: 2019-nCoV, coronavirus, Wuhan, respiratory tract infection, SARS-CoV-2

DOI: 10.7861/clinmed.2019-coron

\section{Introduction}

Over the course of December 2019 in the city of Wuhan in Hubei province, Chinese health authorities identified a cluster of pneumonia cases of unknown aetiology. ${ }^{1}$ Features included pyrexia, radiological signs or acute respiratory distress, reduced or normal white blood cells, lymphopenia, and failure to resolve over 3 to 5 days of antibiotic treatment. Links between the index cases and the city's South China Seafood Market were noted. With the possibility of a new zoonosis or severe acute respiratory syndrome (SARS) outbreak in mind, investigations were undertaken that have since identified a novel coronavirus, SARS-CoV-2 (formerly 2019-nCoV), as the agent now at the heart of an international outbreak centred around Hubei. Despite dramatic headlines in the international press, the outbreak has been characterised by an extraordinarily rapid and effective scientific response, with identification of the pathogen, publication of its genome, and development of highly specific diagnostics within weeks of the initial case detection.

This article will aim to provide a brief review of current knowledge of the COVID-19 acute respiratory disease, and summarise relevant clinical features as currently reported.

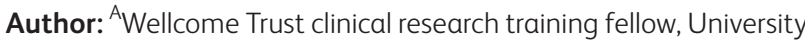
of Cambridge, Cambridge, UK

\section{SARS-CoV-2}

The clinical disease termed COVID-19 is caused by a novel betacoronavirus, now named SARS-CoV-2. SARS-CoV-2 shares $79 \%$ sequence identity with SARS-CoV, the virus which caused a major outbreak in 2002-2003. ${ }^{2-4}$ In common with SARS-CoV, SARS-CoV-2 utilises the ACE-2 receptor for cell entry. Electron microscopy reveals pleomorphic spherical particles, studded with distinctive spike proteins. ${ }^{5}$ Coronaviruses, a family which also includes Middle East respiratory syndrome (MERS) CoV and four of the main agents of the common cold, are zoonotic pathogens. In keeping with this, the first cluster of cases were identified in association with the South China Seafood Market, a 'wet' market at which a large range of live or freshly slaughtered animals were sold including poultry, bats, and snakes. A very early report suggesting a snake host for SARS-CoV-2, such as the Chinese cobra, garnered lurid news headlines but a cold scientific reception. ${ }^{6}$ This report used a technique which exploits the concept that amino acids can be coded for by more than one threenucleotide sequence, and that different species have preferences for particular codon sequences. ${ }^{7}$ These data have since been superseded by more conventional phylogenetic analyses, and SARS-CoV-2 is currently thought to originate from a bat host, based on $88-96 \%$ sequence similarity to bat coronaviruses, in keeping with other pathogenic coronaviruses such as SARS-CoV and MERS-CoV. ${ }^{3,4}$ However, definitive identification of the animal host depends on detection of virus in situ in an infected animal. Additionally, another intermediate animal host more commonly in close contact with humans may also have played a role in Wuhan; SARS-CoV for example is thought to have passed from bats to humans via Himalayan palm civets, Chinese ferret badgers and raccoon dogs sold at the wet markets of Guangdong. ${ }^{8}$

\section{COVID-19 clinical presentation and management}

\section{Clinical features}

The most common symptoms being reported are fever, cough or chest tightness, and dyspnoea. Most cases are reported to experience a mild illness course. ${ }^{9}$ Data regarding the relative frequency of severe illness is likely to be skewed at present by detection bias towards these cases, with sicker patients more likely to present for clinical assessment; these cases may therefore be over-represented in recent data.

Within the subset of patients admitted to hospital, a detailed clinical picture comes from a case series of 41 inpatients with 
laboratory-confirmed COVID-19 disease. ${ }^{10}$ In this cohort, the median age of patients was 49 years (interquartile range (IQR) 41.0-58.0). The most common symptoms at onset of illness were fever $(98 \%)$, cough $(76 \%)$, dyspnoea $(55 \%)$ and myalgia or fatigue $(44 \%)$. Notably, few patients had prominent upper respiratory tract symptoms such as coryza and only one patient had diarrhoea. Other clinical features included sputum production (28\%), headache $(8 \%)$ and, in two cases, haemoptysis. The median time from onset of symptoms to first hospital admission was 7.0 days (IQR 4.0-8.0). Thirty-two per cent required intensive treatment unit (ITU) admission for respiratory support ranging from high-flow nasal cannula to invasive ventilation. Seventy-three per cent of patients were male and $32 \%$ had pre-existing comorbidities such as diabetes (20\%), hypertension (15\%) and cardiovascular disease $(15 \%)$.

Laboratory features in this case series commonly included leukopenia (25\%), lymphopenia (25\%) and raised aspartate aminotransferase (37\%, including seven of 28 non-ITU patients). ITU patients had raised prothrombin and D-dimer levels on admission relative to non-ICU patients. A raised troponin (hypersensitive-troponin I (hs-cTnI)) was detected in five patients, possibly suggestive of virus-associated myocardial injury. Abnormalities on computed tomography (CT) of the chest were seen in all patients, although the indication for imaging was not specified. Ninety-eight per cent had bilateral involvement, with ground-glass opacity and subsegmental areas of consolidation commonly seen. Complications included acute respiratory distress syndrome (29\%) and secondary infection $(10 \%)$.

A follow-up study from the same centre which included these patients and a further 58 cases, found an overall mortality rate of $11 \%$ with $23 \%$ of patients requiring ITU admission, with a preponderance of older males with comorbidities. Half of patients were directly associated with the seafood market, including 47 sales people or market managers. ${ }^{11}$ In an epidemiological study of the first 425 cases in Wuhan, almost half were in adults of 60 years older but, importantly, the case definition at the time specified severe enough illness to require medical attention, thereby potentially missing milder presentations or biasing against younger patients with fewer comorbidities. ${ }^{12}$

\section{Initial assessment}

COVID-19 is classified as an airborne high consequence infectious disease (HCID) in the UK. The cornerstone of management of all possible or confirmed cases is early triage and isolation.

Assessments should be carefully prepared for, including in primary care where many patients are likely to present. During initial assessment, precautions should be taken to minimise transmission including, where possible, initial consultation by telephone. An action plan should be developed in healthcare areas where assessments are likely to occur. The key principles are to identify potential cases as soon as possible; prevent potential transmission of infection to other patients and staff; avoid direct physical contact including physical examination and exposures to respiratory secretions; and to isolate the patient. Practitioners should obtain specialist advice, determine if the patient is at risk of COVID-19, and inform the local health protection team.

Notably, if a history is elicited that suggests a possible case once consultation has already commenced, examination should be abandoned and the practitioner should leave the room, close the door, and wash their hands thoroughly with soap and water. The assessment can then be continued by telephone and the patient triaged appropriately and transfer arranged to an appropriate setting if indicated.

Clinicians are advised to check www.gov.uk/government/ collections/wuhan-novel-coronavirus for the latest triage flowchart.

Prior to assessment of a patient identified as at risk of COVID-19, clinicians must isolate the patient with their belongings and waste in a single occupancy room, preferably a respiratory isolation room and ideally under negative pressure; positive pressure must not be used. Personal protective equipment (PPE) must be worn, comprising, as a minimum, a correctly fitted FFP3 respirator, gown, gloves and eye protection. Practitioners should be trained in the safe putting on and removal of PPE. Patients should be asked to wear a surgical facemask during transport to isolation.

Patients are identified as possible cases by epidemiological and clinical criteria. An up-to-date case definition may be obtained from www.gov.uk/government/collections/wuhannovel-coronavirus. Any further assessment, investigation and management should take place under strict infection control precautions and with close liaison with local infectious diseases and public health teams. ${ }^{14}$

\section{Diagnosis}

Patients satisfying epidemiological and clinical criteria as specified at www.gov.uk/government/collections/wuhan-novel-coronavirus are classified as a possible case. If a patient meets the case definition, clinicians should consult the latest guidance from their public health authority. ${ }^{15}$ Diagnostic sampling must be undertaken only with appropriate infection control precautions and with discussion with local infectious diseases and public health teams. Testing of any samples should take place in a Biological Safety Level 3 laboratory. It is essential to inform the laboratory before sending samples. Early publication of the pathogen genome has allowed rapid development of a reverse transcription polymerase chain reaction (PCR) based test, and whole genome sequencing may also be carried out on positive samples to aid understanding of transmission and mutations. Point-of-care test kits are likely to be made available shortly for home testing. Serological markers have also been identified but are not currently useful for clinical diagnostics. ${ }^{3}$

\section{Specific clinical management}

At present, there are no recommended antivirals for COVID-19 and management is as per best supportive care for any respiratory disease.

Considerations for patients with severe acute respiratory illness include the early use of empirical antimicrobials and neuraminidase inhibitors to cover for alternative (or coexisting) diagnoses. A systematic review of interventions for the management of SARS-CoV patients found no definite benefits for ribavirin, with possible harm due to haemolytic anaemia and impaired liver function (raised alanine aminotransferase), while data on the combination of lopinavir $400 \mathrm{mg}$ with ritonavir 100 mg orally every 12 hours was inconclusive due to study design, despite prior supportive in vitro findings. ${ }^{16}$ However, a randomised controlled trial of lopinavir/ritonavir for COVID-19 has already been initiated in Wuhan. ${ }^{10}$ There was no definite benefit for corticosteroids in SARS-CoV patients as a group, and some 
studies found possible evidence of harm, such as delayed viral clearance, psychosis, diabetes and avascular necrosis. ${ }^{16}$ Evidence from management of MERS-CoV also suggests corticosteroids may delay viral clearance. ${ }^{17}$ However, other indications for corticosteroids such as exacerbation of asthma could potentially supervene. Remdesivir, a novel nucleotide analogue prodrug with activity against MERS-CoV in mouse models has been given to one COVID-19 patient in the USA on a compassionate basis without adverse events, and randomised controlled trials formally investigating its use in COVID-19 infection have already been registered. ${ }^{18-21}$

Detailed guidance intended for the management of COVID-19 cases developing severe acute respiratory illness has been published by the World Health Organization (WHO) and is available online at www.who.int/emergencies/diseases/novelcoronavirus-2019; it is likely to be regularly updated and is therefore not reproduced here.

\section{Transmission}

Human-to-human transmission is now well established for COVID-19, with an $\mathrm{R}_{0}$ (the expected number of secondary cases produced by a single (typical) infection in a completely susceptible population) currently estimated by the $\mathrm{WHO}$ as $1.4-2$. . $^{22}$ For comparison, seasonal flu has a reported median $\mathrm{R}_{0}$ of 1.28 (IQR $1.19-1.37)$, while measles has an $R_{0}$ usually reported as $12-18 .^{23,24}$ However, an $\mathrm{R}_{0}$ must be calculated from imperfect data and in different populations, and estimates are influenced by local variations in susceptibility, efficiency of case detection and infection control responses. Thus, the reported COVID-19 $R_{0}$ may change as further information becomes available.

Most human coronaviruses are transmitted mainly by the respiratory route or via contact with infected secretions. Samples from seven patients (six of whom were seafood peddlers or deliverers at the wholesale market) with severe pneumonia in ITU early in the current outbreak were found to be positive for SARS-CoV-2 in six bronchoalveolar lavage fluid samples and five oral swabs by quantitative PCR and conventional PCR, supportive of a respiratory transmission route. ${ }^{3}$ Virus has also been detected in patient stool samples. ${ }^{19}$ Fomite spread via contaminated surfaces is also probable, based on SARS-CoV. ${ }^{25-27}$ Nosocomial spread is also a significant concern. ${ }^{28}$ The mean incubation period is brief, reported as 5.2 days, with the 95th percentile of the distribution at 12.5 days (95\% confidence interval 9.2-18). ${ }^{12}$ One case documented an incubation period of just 3 days. ${ }^{22}$ However, it is not completely clear yet when a patient becomes infectious within this time frame. Asymptomatic carriage of virus has been documented; in a 10-year-old boy who also showed lung infiltrates on $\mathrm{CT}^{28}$ Asymptomatic transmission of the virus was reported in a cluster of co-workers in Bavaria; however subsequent information refuted this, with the index patient confirming that they already had mild symptoms (myalgia, fatigue and use of an antipyretic analgesic) at the time of transmission. ${ }^{29,30}$ The relative contribution of asymptomatic carriage or transmission (if such can be confirmed) to the overall disease burden remains unclear.

\section{Public health measures}

The evolution of the current outbreak has seen extraordinary measures put in place to control transmission, including the 'shut-down' of Wuhan. Its efficacy or otherwise as a containment measure will become clear over the coming weeks and months. This dramatic action, quarantining a key transport hub 4 hours from Bejing by train, is likely to have huge economic impact, as well as affecting its 11 million people at the individual level.

Screening is currently in place at ports of exit in China, seeking fever above $38^{\circ} \mathrm{C}$ or cough, accompanied in those with symptoms with interview for history of potential exposure to high-risk contacts or to the presumed animal source. The UK, among many other countries, has implemented screening at key entry points. However, as most patients will develop symptoms after travel, a key priority is informing travellers what to do if they do develop symptoms later. At present, travellers entering the UK from Category 1 areas such as Wuhan, Hubei province, Iran, Daegu and Cheongdo in South Korea, or any Italian town under containment measures are requested, even if asymptomatic, to self-quarantine at home for 14 days post travel, and to call NHS 111 for further advice. Travellers from Category 2 areas such as other parts of mainland China and other specified countries (see www.gov.uk for an up-to-date list) are advised to self-quarantine and call NHS 111 if they develop cough, fever or shortness of breath, even if symptoms are mild.

Less dramatically, but no less importantly, the opportunity should be taken to remind patients seeking advice regarding respiratory symptoms to use good cough hygiene: cover their nose and mouth when coughing/sneezing with a tissue or elbow (not hands), immediately dispose of used tissues, and perform hand hygiene after contact with respiratory secretions, on arrival at home from work, school, shopping or leisure activities, before eating and after travel on public transport.

\section{Current outbreak status}

As per the WHO situation report of 04 March 2020, the outbreak stands at 90,870 cases confirmed globally, with 10,566 cases confirmed outside of China in 72 different countries. ${ }^{31}$

\section{Conclusion}

As novel pathogens have emerged and spread, the international response has become successively more sophisticated and far reaching. Initially detected by monitoring systems put in place after the SARS-CoV outbreak of 2002-2003, the emergence of SARS-CoV-2 has been accompanied by a remarkable response from public health bodies and the scientific community. Additionally, major journals and publishers including The Lancet and Elsevier have responded to calls from the Wellcome Trust to make publications relevant to the outbreak immediately available free of charge, promoting wide dissemination of crucial early biological, epidemiological and clinical data. ${ }^{32}$

Although the known severity of COVID-19 disease ranges from mild symptoms of upper respiratory tract infection (with or without fever) to severe pneumonia, most reported cases are at the mild end of the spectrum. At the time of writing, if COVID-19 infection is seen in the UK, it is most likely to occur in travellers that have recently returned from specified countries and areas; or had contact with a known case. Therefore, an accurate history including travel is a key component of risk identification.

\section{Acknowledgements}

Grateful thanks are due to Dr Colin Brown and Prof Cliodna McNulty. 


\section{References}

1 ProMED. PRO/AH/EDR > Undiagnosed pneumonia - China (HU): RFI. ProMED-mail, 2020. https://promedmail.org/promedpost/?id=20191230.6864153 [Accessed 31 January 2020].

2 Wu F, Zhao S, Yu B et al. Complete genome characterisation of a novel coronavirus associated with severe human respiratory disease in Wuhan, China. bioRxiv 2020:[Epub ahead of print].

3 Zhou P, Yang XL, Wang XG et al. Discovery of a novel coronavirus associated with the recent pneumonia outbreak in humans and its potential bat origin. bioRxiv 2020:[Epub ahead of print].

4 Lu R, Zhao X, Li ] et al. Genomic characterisation and epidemiology of 2019 novel coronavirus: implications for virus origins and receptor binding. Lancet 2020:[Epub ahead of print].

5 Zhu N, Zhang D, Wang W et al. A Novel coronavirus from patients with pneumonia in China, 2019. N Engl ] Med 2020:[Epub ahead of print].

6 Callaway E, Cyranoski D. Why snakes probably aren't spreading the new China virus. Nature 2020.

7 Ji W, Wang W, Zhao X, Zai J, Li X. Homologous recombination within the spike glycoprotein of the newly identified coronavirus may boost cross-species transmission from snake to human. J Med Virol 2020:[Epub ahead of print].

8 Guan Y, Zheng BJ, He YQ et al. Isolation and characterization of viruses related to the SARS coronavirus from animals in Southern China. Science 2003:302:276-8.

9 Department of Health and Social Care, Public Health England. Coronavirus: latest information and advice. GOV.UK, 2020. www. gov.uk/guidance/wuhan-novel-coronavirus-information-for-thepublic [Accessed 31 January 2020].

10 Huang $C$, Wang $Y$, Li X et al. Clinical features of patients infected with 2019 novel coronavirus in Wuhan, China. Lancet 2020;6736:1-10.

11 Chen N, Zhou M, Dong X et al. Epidemiological and clinical characteristics of 99 cases of 2019 novel coronavirus pneumonia in Wuhan, China: a descriptive study. Lancet 2020:[Epub ahead of print]

12 Li Q, Guan X, Wu P et al. Early transmission dynamics in Wuhan, China, of novel coronavirus-infected pneumonia. N Engl J Med 2020:[Epub ahead of print].

13 Guan W, Ni Z, Hu Y et al. Clinical characteristics of 2019 novel coronavirus infection in China. medRxiv 2020:[Epub ahead of print].

14 Public Health England. Novel coronavirus (2019-nCoV): infection prevention and control. GOV.UK, 2020. www.gov.uk/government/ publications/wuhan-novel-coronavirus-infection-prevention-andcontrol [Accessed 05 February 2020].

15 Public Health England. Investigation and initial clinical management of possible cases of Wuhan novel coronavirus (2019-nCoV) infection. GOV.UK, 2020 www.gov.uk/government/publications/wuhan-novelcoronavirus-initial-investigation-of-possible-cases/investigationand-initial-clinical-management-of-possible-cases-of-wuhan-novelcoronavirus-wn-cov-infection [Accessed 31 January 2020].

16 Stockman LJ, Bellamy R, Garner P. SARS: Systematic review of treatment effects. PLoS Med 2006:3:1525-31.

17 Public Health England. MERS-CoV: clinical decision making support for treatment. GOV.UK, 2017. www.gov.uk/government/ publications/mers-cov-clinical-decision-making-support-fortreatment [Accessed 07 February 2020].
18 Sheahan TP, Sims AC, Leist SR et al. Comparative therapeutic efficacy of remdesivir and combination lopinavir, ritonavir, and interferon beta against MERS-CoV. Nat Commun 2020;11:222.

19 Holshue ML, DeBolt C, Lindquist S et al. First case of 2019 novel coronavirus in the United States. N Engl J Med 2020:[Epub ahead of print].

20 Mild/Moderate 2019-nCoVRemdesivirRCT. ClinicalTrials.gov, 2020. https://clinicaltrials.gov/ct2/show/NCT04252664 [Accessed 06 February 2020].

21 Severe 2019-nCoVRemdesivirRCT. ClinicalTrials.gov, 2020. https:// clinicaltrials.gov/ct2/show/NCT04257656 [Accessed 10 February 2020].

22 Phan LT, Nguyen TV, Luong QC et al. Importation and human-tohuman transmission of a novel coronavirus in Vietnam. N Engl J Med 2020:[Epub ahead of print].

23 Biggerstaff M, Cauchemez S, Reed C, Gambhir M, Finelli L. Estimates of the reproduction number for seasonal, pandemic, and zoonotic influenza: a systematic review of the literature. BMC Infect Dis 2014:14:480.

24 Guerra FM, Bolotin S, Lim G et al The basic reproduction number (R0) of measles: a systematic review. Lancet Infect Dis 2017;17:e420-8.

25 Xiao S, Li Y, Wong TW, Hui DSC. Role of fomites in SARS transmission during the largest hospital outbreak in Hong Kong. PLoS One 2017;12:e0181558.

26 Dowell SF, Simmerman JM, Erdman DD et al. Severe acute respiratory syndrome coronavirus on hospital surfaces. Clin Infect Dis 2004;39:652-7.

27 Booth TF, Kournikakis B, Bastien N et al. Detection of airborne severe acute respiratory syndrome (SARS) coronavirus and environmental contamination in SARS outbreak units. J Infect Dis 2005;191:1472-7.

28 Chan JF, Yuan S, Kok KH et al. A familial cluster of pneumonia associated with the 2019 novel coronavirus indicating person-toperson transmission: a study of a family cluster. Lancet 2020:[Epub ahead of print].

29 Rothe C, Schunk M, Sothmann P et al. Transmission of 2019-nCoV infection from an asymptomatic contact in Germany. N Engl J Med 2020:[Epub ahead of print].

30 Kupferschmidt K. Study claiming new coronavirus can be transmitted by people without symptoms was flawed. Science, 2020.

31 World Health Organization. Coronavirus disease (COVID-2019) situation reports. Geneva: WHO; 2020. www.who.int/emergencies/ diseases/novel-coronavirus-2019/situation-reports [Accessed 04 March 2020].

32 Carr D. Sharing research data and findings relevant to the novel coronavirus (nCoV) outbreak. Wellcome Trust, 2020. https:// wellcome.ac.uk/press-release/sharing-research-data-and-findingsrelevant-novel-coronavirus-ncov-outbreak. [Accessed 06 February 2020].

Address for correspondence: Dr Alexandra Lake, Laboratory of Molecular Biology, Cambridge Biomedical Campus, Francis Crick Ave, Cambridge CB2 0QH, UK.

Email: alexandra.lake@doctors.org.uk 